\title{
Protostellar Disks, Planet Traps, and the Origins of Exoplanetary Systems
}

\author{
Ralph E. Pudritz ${ }^{1,2}$ and Yasuhiro Hasegawa ${ }^{3}$ \\ ${ }^{1}$ Department of Physics and Astronomy, McMaster University, \\ Hamilton, ON L8S 4M1, Canada \\ ${ }^{2}$ Origins Institute, McMaster University, Hamilton, ON L8S 4M1, Canada \\ email: pudritz@mcmaster.ca \\ ${ }^{3}$ EACOA fellow, Institute of Astronomy and Astrophysics, \\ Academia Sinica (ASIAA), Taipei 10641, Taiwan \\ email: yasu@asiaa.sinica.edu.tw
}

\begin{abstract}
The mass-semimajor axis diagram for exoplanets is populated by at least three distinct planetary populations: hot Jupiters at small orbital radii, more massive Jovian planets gathered at about $1 \mathrm{AU}$, and a rapidly growing population of SuperEarths at short periods. Our work shows that low mass and rapidly migrating planetary cores get trapped at disk inhomogeneities, where strong density or thermal gradients exist (namely dead zone boundaries, ice lines, and disk heating transition regions). Planet growth and movement occur at rates dictated by planetary accretion, and the slow radial inward motion of the traps due to falling disk accretion rates during disk evolution. By combining the theory of traps in evolving disks with standard ideas about how protoplanets accrete, we develop evolutionary tracks of how planets evolve in the mass- semimajor axis diagram. Our models account for the planetary "pile-up" at $1 \mathrm{AU}$, the origin of SuperEarths and hot Jupiters, and the relative scarcity of Jovian planets at large distances.
\end{abstract}

Keywords. accretion disks, (stars:) planetary systems: formation, (stars:) planetary systems: protoplanetary disks.

\section{Introduction}

Arguably the most important means that we have for exploring the origin of exoplanets and planetary systems is the mass-semimajor axis diagram. The discovery of large numbers of exoplanets by various kinds of surveys over the last decade reveals that several distinct kinds of planetary populations exist in this diagram (see Chiang \& Laughlin 2013). The original discovery of the population of hot Jupiters at a relative orbit of Mercury and masses that are somewhat subJovian (denoted as Zone 1) has been augmented by several other populations: a region that is quite depleted of gas giants (Zone 2), more massive Jovian planets gathered around a semimajor orbital axis of 1 AU (Zone 3), an exciting new population of imaged young Jovian planets at large orbital radii $a>10 A U$ (Zone 4), and a strongly emerging population of SuperEarths of mass $1-10 M_{E}$ and hot Neptunes close into their host stars (Zone 5). We highlight these populations in Figure 1 (Hasegawa \& Pudritz (2013)). The location of planets in this diagram is largely a consequence of the formation and migration of planets in protostellar disks.

Theories of planet-disk interaction suggest that many parameters may control the migration and growth of planets in disks. In the context of the core-accretion model for planet formation, the population synthesis approach is often used to try to match populations seen in the mass-semimajor axis diagram. By varying a large number of disk parameters in this Monte Carlo approach, it was shown that Type I migration in 


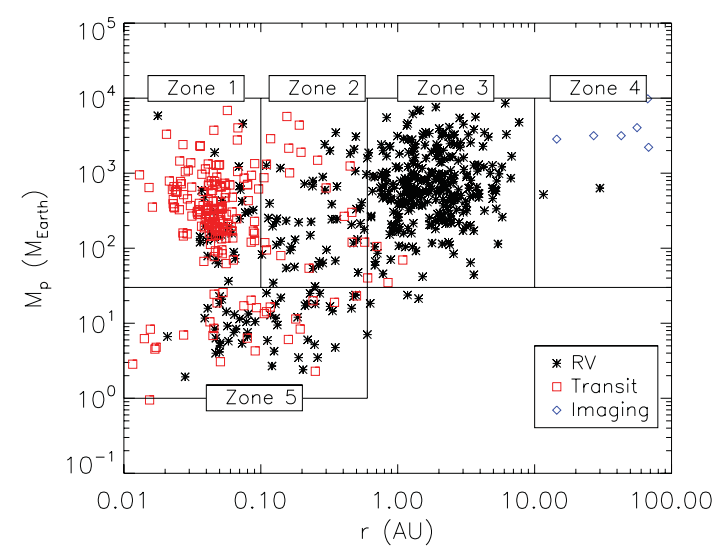

Figure 1. Mass - semimajor axis relation; data taken from exoplanets.eu. Planets observed by RV technique, stars; transits, squares; and direct imaging, diamonds. We denote Zone 1 - hot Jupiters, Zone 2 - exclusion of gas giants, Zone 3 - pile up of gas giants, Zone 4 - distant Imaged planets, and Zone 5 - SuperEarths and hot Neptunes. Adapted from Hasegawa \& Pudritz (2013)

homogenous disks occurs far too rapidly $\left(\sim 10^{5}\right.$ years for planets with $\sim 1 M_{\oplus}$ at $\left.\sim 1 \mathrm{AU}\right)$ to account for the data ( eg. Ida \& Lin 2008, Mordasini et al. 2009 ). A physical solution to this dilemma is that disks are quite inhomogenous structures and that rapidly migrating planetary cores become trapped in narrow regions with marked changes in density and temperature, - such as dead zone boundaries, ice lines, and heat transitions - that are regions of net zero torque ( Masset et al. (2006), Hasegawa \& Pudritz 2011). Planet traps, in turn, move to smaller disk radii as the disk accretion rate falls, which is on a several Myr timescale. The question then is - what kinds of evolutionary tracks connect progenitor protoplanetary cores, born and developed on their host planet traps, to their final positions in this diagram? We show that the combination of this slower radial inward movement of traps in evolving disks, coupled with the standard picture of accretion onto protoplanetary cores, provides an excellent explanation of these populations.

\section{Evolutionary Tracks in the Mass-Semimajor Axis Diagram}

The growth in mass of an accreting planetary core can be combined with the radial inward motion of its host planet trap in its evolving disk, to create a unique evolutionary track in the mass-semimajor axis diagram. Analytic solutions of the motion of the traps were worked out in Hasegawa \& Pudritz 2012 (see also Hasegawa \& Pudritz, these Proceedings). The three types of traps have different power law dependences on the mass accretion rate through the disk. The disk accretion rate is in turn driven by a combination of disk viscosity (cf. Lynden-Bell \& Pringle 1974), which gradually clears out disk mass onto the star over some accretion time scale, coupled with the eventual rapid loss of the disk due to photoevaporation (Adams et al. 2004). We therefore model this process as depending upon two key parameters: the dimensionless disk accretion rate $\eta_{a c c}$ (in units of a fiducial TTS accretion rate $3 \times 10^{8} M_{\odot} y r^{-1}$ ), and the dimensionless disk depletion time $\eta_{d e p}$ (in units of a fiducial disk lifetime, or depletion time, of $\simeq 10^{6} \mathrm{yr}$ ).

The increase in planetary mass due to accretion is well known to follow 3 stages (e.g. Pollack et al. 1996): the formation of rocky cores through runaway and oligarchic growth (Stage I) lasting $\simeq 10^{5} \mathrm{yr}$, the subsequent slow gas accretion of the cores and formation of envelopes surrounding them (Stage II) lasting $\simeq 10^{6} \mathrm{yr}$, and the rapid collapse of the envelopes and runaway gas accretion onto their cores once the core mass exceeds a critical 

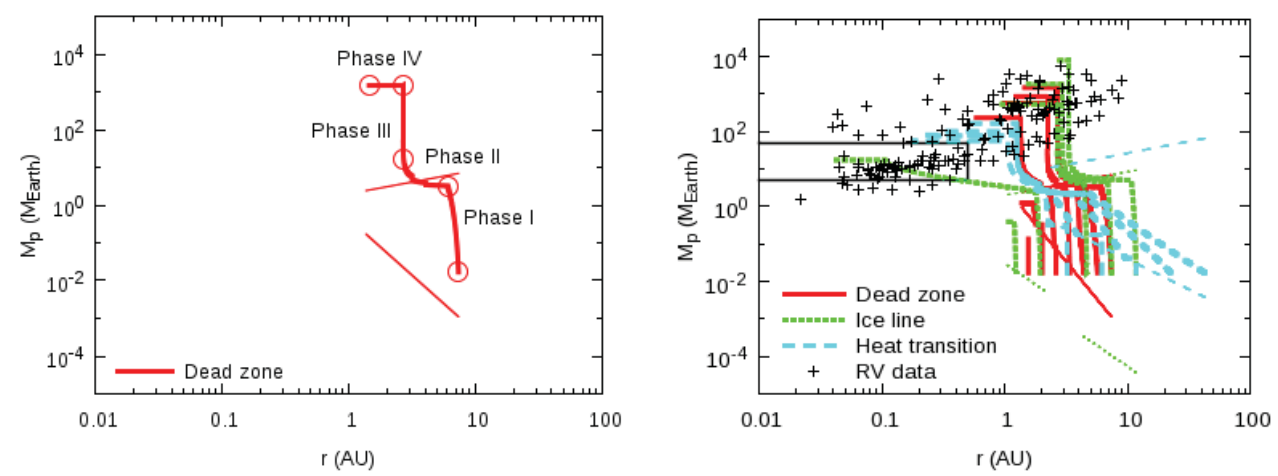

Figure 2. (a) Evolutionary track for an initial seed core being pulled along through the disk by a dead zone trap; (b) full set of tracks for the 3 types of planet traps; dead zones, the water ice line, and the heat transition. Adapted from Hasegawa \& Pudritz 2012.

mass $M_{c r}$ of the order of a few Earth masses (Stage III). In order to model these three physical processes, we adopt the formulation of Ida \& Lin (2004). The whole accretion process may take a few Myr to complete.

In Figure 2(a) we show a typical evolutionary track of a small initial progenitor core (hundredth of an Earth mass) as it accretes and is pulled to smaller disk radii by a dead zone trap. The evolution undergoes 4 recognizable phases. In Phase I, runaway oligarchic growth of the core occurs on a much faster time scale than the disk, and trap, evolution time of $10^{6} \mathrm{yr}$. Hence the track proceeds in a strongly vertical direction of increasing mass with only a slight inward shift of orbital radius. In phase II, slow gas accretion dominates over a long time span so that the track barely ascends in mass as the dead zone trap moves to smaller disk radii. In phase III, the core has reached its critical value and the planet accretes most of its mass very rapidly through runaway gas accretion hence the extensive and nearly vertical motion in the diagram with little radial inward motion of the trap. At the end of this phase, the planet has achieved its gap opening mass and ceases to accrete. It now enters a slower, Type II migration phase wherein the planet migrates inwards with no gas accretion. This phase ends when the disk is finally dispersed by photoevaporation (Hasegawa \& Pudritz (2012)). In Figure 2(b) we show a whole collection of tracks calculated for initial small cores distributed at different initial disk radii and carried along by the three respective traps. The tracks cover all of the populations in the earlier data set (based only on RV measurements), including a region (rectangle) that was claimed to be a "planetary desert" in the Ida and Lin picture.

\section{Planet Formation Frequencies and Planetary Populations}

We computed a large number of tracks (several hundred) for a population of disks that are controlled by just two parameters: the dimensionless disk accretion rate $\eta_{a c c}$ and disk depletion time $\eta_{d e p}$ (Hasegawa \& Pudritz (2013). We define the planet formation frequency $(\mathrm{PPF})$ for each zone as the fraction of the total tracks leading to zone $i$, integrated over all values of these two variables. Planets formed in different traps populate different types of target zones: Jovian planets around 1AU are largely fed by dead zone and ice line traps, whereas the closer in populations are fed by a combination of dead zone and heat transition traps. The PPFs of hot Jupiters are $1.6 \%$, those of gas giants around $1 \mathrm{AU}$ are $24 \%$ (the largest populations), and those of low-mass planets in tight orbits are $15 \%$. The SuperEarths are well explained as being failed cores in the longer 


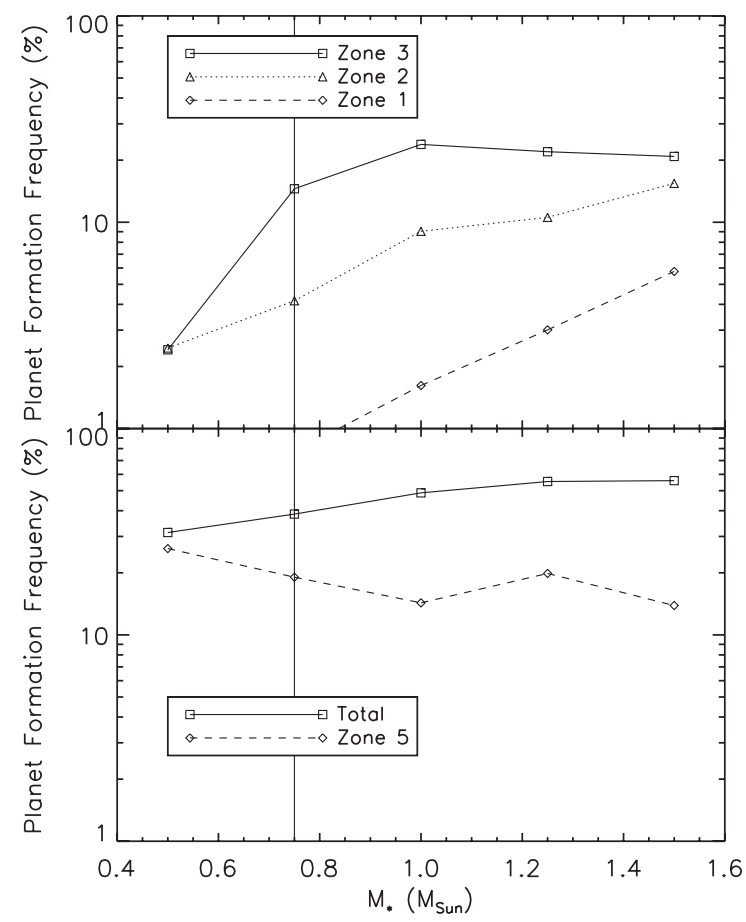

Figure 3. Planet Formation Frequencies (PPFs) - populations in the mass-semimajor axis diagram as a function of stellar mass. Adapted from Hasegawa \& Pudritz 2013.

lived disk populations. Notably, there are only a few tracks that connect to giant planets at large radii, which agrees with the results of the NICI imaging surveys (see Vigan et al., these proceedings) that find few objects beyond 10 AU. Finally, we show the PPFs for each zone, for stars of masses $0.5-1.5 M_{\odot}$ in Figure 3. The low-mass planets in tight orbits dominate the planetary population for low mass stars $\left(M \leqslant 0.7 M_{\odot}\right)$, while massive Jupiters (zone 3) dominate for larger stellar masses, in good agreement with the observations.

\section{References}

Adams, F. C., Hollenbach, D., Laughlin, G., \& Gorti, U.2004, ApJ, 611, 360

Chiang, E. \& Laughlin, G.2013, MNRAS, 431, 3444

Hasegawa, Y. \& Pudritz, R. E. 2011, MNRAS, 417, 1236

Hasegawa, Y. \& Pudritz, R. E. 2012, ApJ, 760, 117

Hasegawa, Y. \& Pudritz, R. E. 2013, ApJ, 778, 78

Ida, S. \& Lin, D. N.C. $2004, A p J, 604,388$

Ida, S. \& Lin, D. N.C. 2008, ApJ, 673, 487

Lynden-Bell, D. \& Pringle, J. E.1974, MNRAS, 168, 603

Masset, F. S., et al. 2006, ApJ, 642, 478

Mordasini, C., Alibert, Yl, \& Benz, W.2009 AESA, 501, 1139

Pollack, J. B., Hubickyj, O., Bodenheimer, P. et al.,1996 Icarus,124, 62

\section{Discussion}

JACKSON: It looks like you may be forming to many planets in your 'exclusion zone' ( 0.1-1 AU) compared to those at 1-10 AU, amd maybe compared to the hot Jupiters at $<0.1$ AU. Do you want to comment on that? 
PUDRITZ: If you look carefully at our predictions for the total number of Zone 1 (hot Jupiters) vs. Zone 2 ("empty zone") there is a factor of 2-3 higher for Zone 1. This seems to agree with the observations.

TinNeY: How will planet multiplicity impact on your conclusions? It appears you could have situations where Super Earths are required to cross gas giant orbits in order to get to where we find them.

PUDRITZ: Planet traps tend to converge and sometimes intersect at late times in disk evolution. Therefore we would expect that under those circumstances, planet-planet interaction will become very important.

Macintosh: I should note that the statistical power of direct imaging surveys is much lower than Doppler, so the number of planets from say 10-50 AU could actually still be similar to $0.2-1 \mathrm{AU}$.

PUDRITZ: Interesting point. We will continue to run our models, but at the moment it still appears that it will be hard to populate the distant regions ( $>30 \mathrm{AU})$. We are eager to see the new data. 